SRL-ETS-90-293

\title{
OFFSITE DOSES FROM SRS RELEASES OVER THE PERIOD OF SITE OPERATION (U)
}

by L. R. Bauer

Westinghouse Savannah River Company

Savannah River Site

Aiken, South Carolina 29808

Other Authors:
SRL-ETS--90-293

DE93 005521

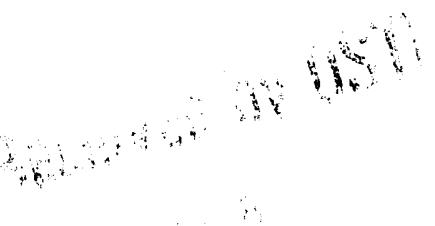

This paper was prepared in connection with work done under Contract No. DE-AC09-89SR18035 with the U.S. Department of Energy. By acceptance of this paper, the publisher and/or recipient acknowledges the U.S. Government's right to retain a nonexclusive, royalty-free license in and to any copyright covering this paper, along with the right to reproduce and to authorize others to reproduce all or part of the copyrighted paper. 


\section{DISCLAIMER}

This report was prepared as an account of work sponsored by an agency of the United States Government. Neither the United States Government nor any agency thereof, nor any of their employees. makes any warranty, express or implied, or assumes any legal liability or responsibilicy for the accuracy, completeness, or usefulness of any information, apparatus, product, or process disclosed, or represents that its use would not infringe privately owned rights. Reference herein to any specific commercial product, process, or service by trade name, trademark, manufacturer, or otherwise does not necessarily constitute or imply its endorsement, recommendation, or favoring by the United States Government or any agency thereof. The views and opinions of authors expressed herein do not necessarily state or reflect those of the United States Government or any agency thereof.

This report has been reproduced directly from the best available copy.

Available to DOE and DOE contractors from the Office of Scientific and Technical Information, P.O. Box 62, Oak Ridge, TN 37831; prices available from (615) 576-8401, FTS 626-8401.

Available to the public from the National Technical Information Service, U.S. Department of Commerce, 5285 Port Royal Rd., Springfield, VA 22161. 


\title{
SAVANNAH RIVER LABORATORY DIVISION ENVIRONMENTAL TECHNOLOGY SECTION
}

July 13,1990

TO: C.B. Shedrow, 773-42A

FROM: LR. Bauer, 773-A LRB

REF: Questions Generated by RO EIS Public Hearings

\author{
Offsite Doses from SRS Releases \\ Over the Period of Site Operation
}

Doses to offsite individuals and at-risk populations from SRS releases for the period 1954 - 1989 have been estimated. These data have been generated in response to questions regarding the overall impact of SRS operations on the offsite environment. The data are comprehensive in the sense that all known measuredand calculated atmospheric and liquid release values (Tables 1 and 2, respectively) have been included in the assessment.

This work should not be interpreted as a formal dose reconstruction as current sitespecific data (Table 3) have been used. Also, three key assumptions were required for these doses which affect their interpretation: (1) persons in the at-risk populations were assumed to have remained at their designated locations continuously from 1954 - 1989, (2) it was assumed that these individuals will live for 50 additional years following 1989, and (3) the Beaufort-Jasper water treatment plant was assumed to have operated continually since 1954 even though operations actually began in 1965 . The values reported in this memorandum are therefore believed to be bounding cases which ultimately will be shown to have significantly overestimated the doses.

Dose estimates for several at-risk individuals and populations are listed in Table 4 . A comparison of the doses from the SRS and other sources of ionizing radiation in the environment has also been included. As Table 4 indicates, SRS releases from 1954 - 1989 appear to have presented negligible additional risks for individuals and populations in the vicinity of the SRS.

cc: A.L. Boni, 773-A

D.M. Hamby, 773-A

H.D. Harmon, 773-A

W.L. Marter, 773-42A

SRL Records (2)

EDG Files (1) 
Table 1. Cumulative Atmospheric SRS Releases, Ci (1954 - 1989).

\begin{tabular}{|c|c|c|c|c|c|c|}
\hline Radionuclide & $\begin{array}{r}1954 \\
-1985 \\
\end{array}$ & 1988 & 1987 & 1988 & 1089 & $\begin{array}{l}\text { Value Used } \\
\text { in Dose } \\
\text { Evaluation (c) }\end{array}$ \\
\hline Total H-3 (a) & $2.27 E+07$ & & & & & \\
\hline HTO+0.004\%HT & & $2.85 E+05$ & $2.70 E+05$ & $2.88 E+05$ & $2.18 E+05$ & $2.38 E+07$ \\
\hline C-14 & $2.84 E+03$ & $5.60 E+01$ & $4.10 E+01$ & $2.40 E+01$ & $1.80 E+01$ & $2.98 E+03$ \\
\hline$A R-41$ & $6.16 E+06$ & $8.32 E+04$ & $8.77 E+0.4$ & $2.95 E+04$ & & $6.36 E+06$ \\
\hline $10-60$ & 9.15E-02 & $8.00 E-06$ & $1.30 E-05$ & $3.00 E-06$ & & $9.15 E-02$ \\
\hline SE-75 & $1.06 E-04$ & 2.10E-05 & 4.00E-04 & 2.00E-05 & & $5.47 E-04$ \\
\hline KR-85 (b) & $9.22 E+06$ & $7.10 E+0.5$ & $3.95 E+05$ & $2.40 E+05$ & $4.17 E+04$ & $1.06 E+07$ \\
\hline $\mathrm{KR}-85 \mathrm{~m}$ & $1.13 E+05$ & $1.99 E+03$ & $1.69 E+03$ & $1.02 E+03$ & & $1.18 E+05$ \\
\hline KR-87 & $1.62 E+04$ & $1.38 E+03$ & $1.16 E+03$ & $1.36 E+03$ & & $2.01 E+04$ \\
\hline KR-88 & 8.77E+04 & $2.43 E+03$ & $2.01 E+03$ & $1.61 E+03$ & & $9.38 E+04$ \\
\hline SR-89-90 & $1.53 E+00$ & 1.97E-03 & $1.35 E-03$ & $3.01 E-03$ & $1.14 E-03$ & $4.70 E+01$ \\
\hline ZR.95 & 5.51E-01 & 4.38E-03 & $1.67 E-03$ & 7.6OE-04 & $5.80 E-05$ & $3.12 E+\infty 0$ \\
\hline NB-95 & $1.06 E+\infty 0$ & $9.18 E-03$ & $3.29 E-03$ & 2.22E- -03 & $1.28 E-04$ & $1.08 E+\infty 0$ \\
\hline ZR-NB-95 & $2.56 E+\infty$ & & & & & (Zr-95) \\
\hline RU-103 & $1.91 E+01$ & $3.50 E-03$ & $1.37 E-03$ & 4.61E-04 & $7.00 E-06$ & $1.91 E+01$ \\
\hline RU-106 & $4.58 E+01$ & 5.90E-02 & 4.53E-02 & 3.02E-02 & $3.26 E-03$ & $1.39 E+02$ \\
\hline RU-103-106 & $9.31 E+01$ & & & & & $(R u-106)$ \\
\hline $1-129 \ldots$ & $5.40 E+\infty 0$ & 8.70E-02 & $7.20 E-02$ & 6.30E-02 & 5.19E-02 & 5.67E $+\infty 0$ \\
\hline$|-13|$ & $2.52 E+03$ & 2.64E-02 & $1.26 E-02$ & 4.96E-04 & $3.64 E-04$ & $2.52 E+03$ \\
\hline $\mid-133 \ldots$ & $1.02 E-02$ & & & & & $1.02 E-02$ \\
\hline XE-131m & $1.01 E+04$ & 3.00E-01 & & & & $1.01 E+04$ \\
\hline XE-133 & $4.52 E+05$ & $1.06 E+04$ & $5.32 E+03$ & $6.97 E+03$ & & $4.75 E+05$ \\
\hline$X E-135$ & $1.51 E+05$ & $2.60 E+03$ & $3.48 E+03$ & $2.98 E+03$ & & $1.60 E+05$ \\
\hline CS-134 & $9.04 E-03$ & 6.94E-04 & 2.20E-03 & $1.00 E-04$ & 9.20E-05 & 1.21E-02 \\
\hline CS-137 & $2.43 E+00$ & $2.95 E-03$ & $1.07 E+00$ & $1.78 E-03$ & 9.56E-04 & $3.50 E+\infty 0$ \\
\hline CE-141 & 4.60E-02 & $1.90 E-05$ & $6.00 E-06$ & 1.60E-05 & $1.00 E-06$ & 4.60E-02 \\
\hline CE-144 & 7.20E-01 & $1.10 E-02$ & $3.15 E-02$ & $4.56 E-03$ & 7.62E-04 & $4.17 E+\infty 0$ \\
\hline CE-141-144 & $3.40 E+\infty 0$ & & & & & $(C 0-144)$ \\
\hline OS-185 & 1.53E-03 & $1.40 E-04$ & $7.00 E-05$ & $3.00 E-05$ & & 1.77E-03 \\
\hline U-238 & 8.37E-01 & 1.57E-03 & 8.52E-03 & $1.47 E-03$ & $5.05 E-03$ & 8.54E-01 \\
\hline PU-238 & 6.93E-01 & 2.02E-03 & $1.96 E-03$ & $6.16 E-04$ & 8.61E-04 & $6.98 E-01$ \\
\hline PU-239 & $3.02 E+\infty 0$ & $3.36 E-04$ & 4.07E-04 & 6.87E-04 & $1.30 E-03$ & $3.10 E+\infty 0$ \\
\hline$A M-241,243$ & 4.81E-03 & $1.54 E-04$ & $3.22 E-04$ & 6.70E-05 & $2.01 E-04$ & $5.56 E-03$ \\
\hline$C M-242$ & 2.34E-03 & & & & & 2.34E-03 \\
\hline CM-244 & 8.90E-02 & & & & & 8.94E-02 \\
\hline CM-242,244 & & 2.80E-05 & 2.04E-04 & $1.18 E-04$ & $2.80 E-05$ & (Cm-244) \\
\hline UNID-ALPHA & 7.55E-02 & & & & & (Pu-239) \\
\hline UNID-BETA+GAMMA & $4.54 E+01$ & & & & & $(\mathrm{Sr}-89,90)$ \\
\hline
\end{tabular}

(a) Data on relative elemental and oxide release rates are not available for $1954-1985$. Releases during that period were assumed to be exclusively tritium oxide. This assumption significantly overestimates doses.

(b) Kr-85 release data prior to 1970 are classifled and cannot be included in this report.

(c) Symbols in parentheses indicate the radionuclides to which non-specific source terms were assigned. Such assignments were based on comparable yet conservative transport and dose conditions. 
Table 2. Cumulative Liquid SRS Releases, Cl (1954 - 1989).

\begin{tabular}{|c|c|c|c|c|c|c|c|}
\hline \multirow[b]{3}{*}{ Radionuclide } & \multicolumn{2}{|c|}{$1954-1985$} & \multirow{2}{*}{\multicolumn{4}{|c|}{ Direct Releases + SB Migration }} & \multirow{3}{*}{$\begin{array}{l}\text { Value Used } \\
\text { in Dose } \\
\text { Evaluation (a) }\end{array}$} \\
\hline & \multirow{2}{*}{$\begin{array}{l}\text { Direct to } \\
\text { Streams }\end{array}$} & \multirow{2}{*}{$\begin{array}{c}\text { S Basin } \\
\text { Mlgration }\end{array}$} & & & & & \\
\hline & & & 1986 & 1987 & 1988 & 1989 & \\
\hline $\mathrm{H}-3$ & $1.44 E+06$ & $3.80 E+05$ & $2.8 E+04$ & $2.3 E+04$ & $1.93 E+04$ & $1.74 E+04$ & $1.91 E+06$ \\
\hline P-32 & $3.64 E+01$ & & & & & & $3.64 E+01$ \\
\hline S-35 & $1.47 E+03$ & & & & & & $1.47 E+03$ \\
\hline CR-51 & $4.48 E+03$ & & & & & & $4.48 E+03$ \\
\hline MN-54 & 6.41E-01 & & & & & & $6.41 E-01$ \\
\hline CO-60 & $6.64 E+01$ & & & & & & $6.64 E+01$ \\
\hline $\mathrm{ZN}-65$ & $1.42 E+02$ & & & & & & $1.42 E+02$ \\
\hline SR-89 & $2.16 E+02$ & & & & & & (Sr-90) \\
\hline SR-90 & $1.03 E+02$ & $1.24 E+01$ & $3.6 E-01$ & 4.0E-01 & 3.77E-01 & 2.89E-01 & $3.78 E+02$ \\
\hline SR-89-90 & 5.88E-02 & & & & & & (Sr-90) \\
\hline$Y-91$ & $1.16 E+02$ & & & & & & $1.16 E+02$ \\
\hline ZR-NB-95 & $1.27 E+02$ & & & & & $1.40 E-05$ & $1.27 E+02$ \\
\hline MO-99 & $2.29 E+01$ & & & & & & $2.29 E+01$ \\
\hline RU-103-106 & $5.86 E+01$ & & & & & $4.61 E-03$ & $5.86 E+01$ \\
\hline SB-124 & $7.54 E+00$ & & & & & & $7.56 \mathrm{E}+0 \sigma$ \\
\hline SB-125 & $4.10 E-04$ & & & & & & $4.10 E-04$ \\
\hline SB-124,125 & 2.29E-02 & & & & & & $(S b-124)$ \\
\hline $1-129$ & $1.30 E-01$ & & $2.2 E-02$ & 2.2E-02 & 2.22E-02 & 2.20E-02 & $2.18 E-01$ \\
\hline $\mid-131$ & $3.02 E+02$ & & & & & & $3.02 E+02$ \\
\hline CS-134 & 5.47E-01 & & & & & & $5.54 E-01$ \\
\hline CS-137 & $6.06 E+02$ & & 1.1E-01 & 3.8E-01 & 5.65E-01 & $2.06 E-01$ & $6.08 E+02$ \\
\hline CS-134-137 & $6.92 E-03$ & & & & & & (Cs-134) \\
\hline BA-LA-140 & $2.22 E+02$ & & & & & & $(B a-140)$ \\
\hline CE-141-144 & $3.50 E+02$ & & & & & 4.50E-05 & $3.50 E+02$ \\
\hline PM-147 & $1.10 E+02$ & & & & $1.97 E-02$ & $3.29 E-04$ & $1.10 E+02$ \\
\hline U-238 & $2.46 E+01$ & & $4.4 E-02$ & 5.5E-03 & $5.51 E-03$ & $4.25 E-04$ & $2.47 E+01$ \\
\hline PU-238 & $1.03 E-01$ & & & & & & $1.03 E-01$ \\
\hline PU-239 & & & 8.5E-03 & $1.8 E-02$ & 5.54E-03 & $1.42 E-02$ & 5.21E-01 \\
\hline NP.239 & $1.43 E+03$ & & & & & & $1.43 E+03$ \\
\hline CM-244 & $3.86 E-01$ & & & & & & $3.86 E-01$ \\
\hline UNID-ALPHA & 4.75E-01 & & & & & & (Pu-239) \\
\hline UNID-BETA+GAMMA & $4.49 E+01$ & & & & & & $(\mathrm{Sr}-90)$ \\
\hline
\end{tabular}

(a) Symbols in parentheses indicate the radionuclides to which non-specific source terms were assigned. Such assignments were based on comparable yet conservative transport and dose conditions. 
Table 3. Environmental Dosse Assessment: Parameter Specifications

Release Period: 1954 - 1989

Atmospheric Releases

Maximum Individual

$\frac{\text { Sector }}{\text { East }} \quad \frac{\text { Distance, mi }}{9.03}$

Population At Risk

80-km Population

$\frac{\text { Size }}{555100} \quad \frac{\text { Composition }}{\text { Adult }}$

Codes: XOQDOQ/GASPAR

Release Heiǵht:

$200 \mathrm{ft}$

Dose Factors: DOE/ICRP 30

Release Point: SRS Center

Met Data:

H-Area $1982-86$

\section{Liquid Releases}

Maximum Individual

80-km Population

Beaufort-Jasper

Port Wentworth

Salt water imvertebrate consumers

Code:

LADTAP \|

Dose Factors: DOE/ICRP 30

\section{Locations Evaluated}

Below SRS

Downstream Water Treatment Plant

$\begin{array}{ll}\frac{\text { Size }}{555100} & \frac{\text { Composition }}{\text { Adult }} \\ 51000 & \text { Adult } \\ 20000 & \text { Adult } \\ 555100 & \text { Adult }\end{array}$

River Flow Rate: $\quad 10000$ cfs
283

$\mathrm{m}^{3} / \mathrm{s}$ 


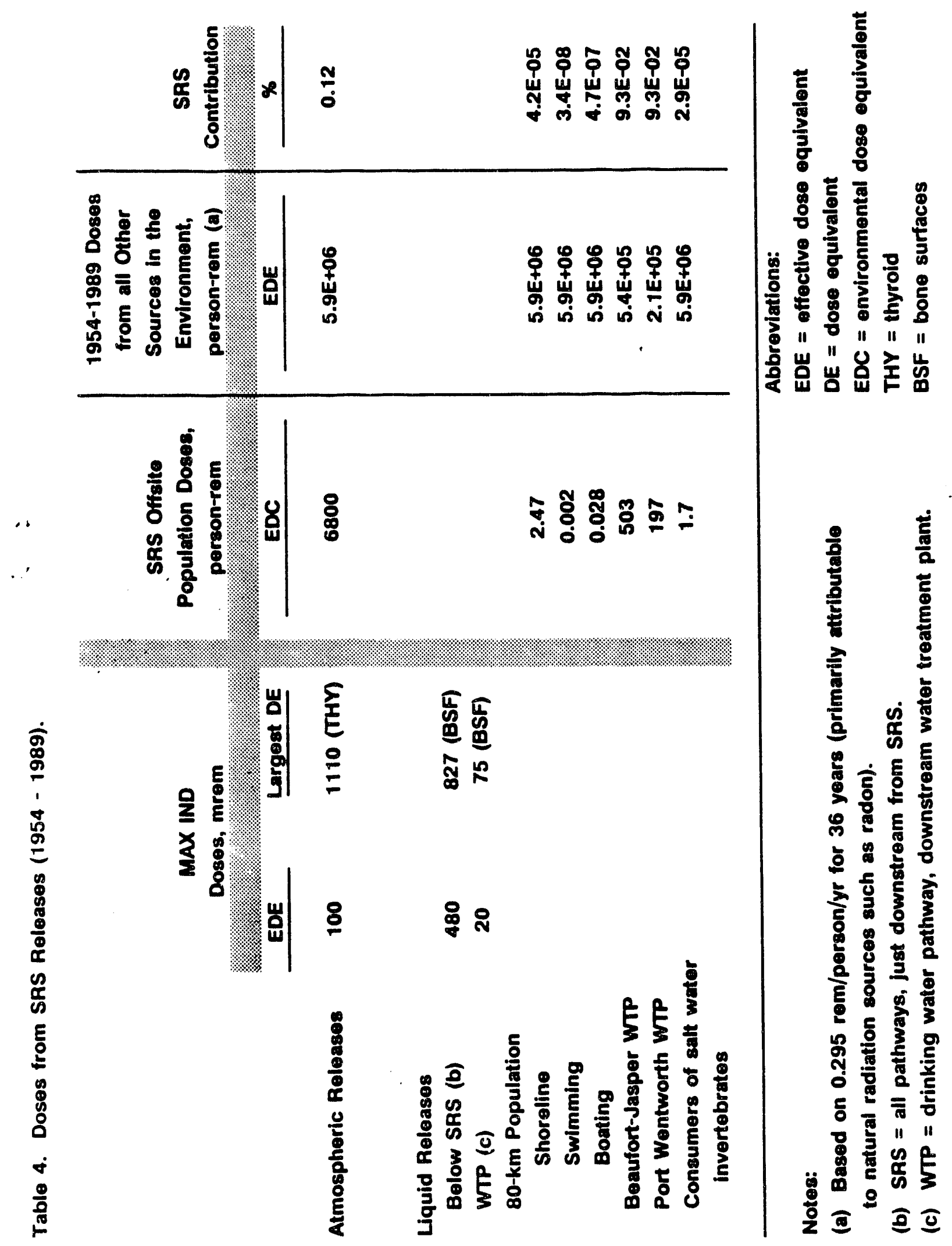




\section{REFERENCES}

H.A. Davis, D.K. Martin, and J.L. Todd. Savannah River Site Environmental Report for 1988. WSRC-RP-89-59-1, Vol. I, Savannah River Site, Aiken, SC 29808 (1990).

W.L. Marter. Offsite Doses for 1989 Environmental Report. SRL-ETS-90-151, Savannah River Site, Aiken, SC 29808 (1990).

S.C. Mikol, L.T. Burckhalter, J.L. Todd, and D.K. Martin. Savannah River Plant Environmental Report - Annual Report for 1987. DPSPU-88-30-1, Vol. I, Savannah River Plant, Aiken, SC 29808 (1988).

C.C. Zeigler, E.M. Heath, L.B. Taus, and J.L. Todd. Savannah River Plant Environmental Report - Annual Report for 1986. DPSPU-87-30-1, Vol. I, Savannah River Plant, Aiken, SC 29808 (1987).

C.C. Zeigler and I.B. Lawrimore. Releases of Radioactivity at the Savannah River Plant. DPSPU-86-25-1, Savannah River Plant, Aiken, SC 29808 (1988). 

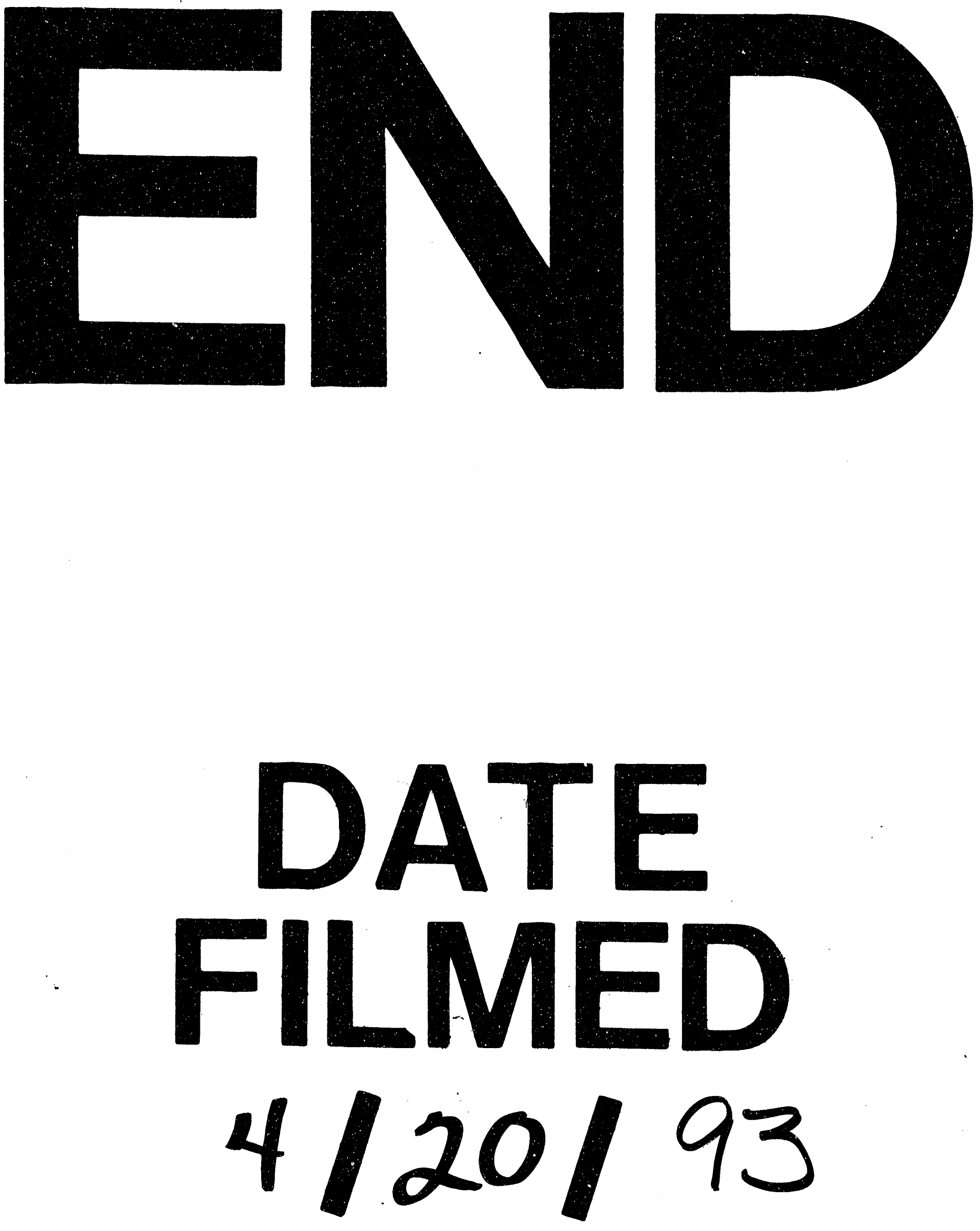
